\title{
Environmental destruction as (objectively) uneventful and (subjectively) irrelevant
}

\section{Ollinaho, Ossi livari}

2016-02-09

Ollinaho , O I 2016 , ' Environmental destruction as (objectively) uneventful and (subjectively) irrelevant ' , Environmental Sociology , vol. 2 , no. 1 , pp. 53-63 . https://doi.org/10.1080/23251042.201

http://hdl.handle.net/10138/302506

https://doi.org/10.1080/23251042.2015.1114207

acceptedVersion

Downloaded from Helda, University of Helsinki institutional repository.

This is an electronic reprint of the original article.

This reprint may differ from the original in pagination and typographic detail.

Please cite the original version. 


\section{Environmental destruction as (objectively) uneventful and (subjectively) irrelevant}

O. I. Ollinaho

This text is Author's Accepted Manuscript that was published in 2016 in Environmental Sociology, Vol. 2, No. 1, 53-63, http://dx.doi.org/10.1080/23251042.2015.1114207

This essay contributes to the recent criticism against individualism and cognitivism in environmental social scientific theorizing by conceptualizing two undertheorized phenomena related to environmental changes: uneventfulness and irrelevance. Under much of environmental predicament lies a process characterized by recurrent acts through which pieces of sociomateriality are taken from or added to a particular sociomaterial system. Such process not only produces cumulative sociomaterial changes, but also the corresponding withdrawals and additions lose their eventfulness over time. Elaborating on Theodore Schatzki's work, complexity is built into the concept of practice rather than accounting for complexity with the interrelatedness of practices. Another part of the essay analyzes the subjectivity of the actor, focusing on the relevance structures of the ordinary citizens in the Global North. Environmental changes are imposed to the consciousness as intellectual problems, which tend to be incommensurate with the pragmatic necessities of everyday life. Applying Alfred Schütz's phenomenological sociology to environmental phenomena opens up new avenues for empirical environmental sociology. Understanding the objective uneventfulness and subjective irrelevance associated with much of environmental changes helps explain the inactivity of the masses amid widespread attention on the environmental predicament. 


\section{Introduction}

This paper scrutinizes at the level of social practice how "we cause environmental problems collectively as participants to global industrial market society ... as a globally dispersed and often socially unequal mass of actors" (Arponen 2013, 47-48), that is, how environmental changes are being produced through the "normal workings" of industrial societies (Rudel, Roberts, and Carmin 2011, 221). Building on Alfred Schütz's (e.g. Schutz 1962, 1964) and Theodore Schatzki's (e.g. Schatzki 1996, 2010) work, the points of departure for this essay are the direct experience of the differentiated individuals in the global system and the ways their actions hang together. The corpus of environmental changes is in nature cumulative; it is made of recurrent additions to or withdrawals from, to use Allan Schnaiberg's (1980) terms, natural or built environments through the reproduction of more or less established practices. Such a cumulative change underlies much of the environmental social theorizing, but has remained unarticulated at the level of social practices. Another phenomenon closely looked at in this essay is the often implied issue that most environmental changes are intrinsically irrelevant for the lay people in their paramount reality; "the subuniverse of the sensorily perceivable, physical world" (Schutz and Luckmann 1973, 5). If environmental sociology aspires to answer the late Ulrich Beck's question, "Why is there no storming of the Bastille because of the environmental destruction threatening mankind" $(2010,254)$, we have to understand the objective uneventfulness of environmental changes and relevance structures of the differentiated masses.

Neither uneventfulness nor irrelevance has been placed under a systematic analytical scrutiny to date even though such phenomena underlie an increasing part of the environmental literature. Frederick Buttel distinguished between two general categories of environmentally related phenomena: "intentionally-environmental" and "unintentionally-environmental" (1996). The former "consists of behaviors or institutional patterns that are self-consciously environmental or environmentally relevant" and the latter "consists of 'ordinary' social practices and phenomena that have environmental dimensions or implications, although they remain invisible or unrecognized" (Buttel 1996, 66-67). Nearly two decades ago Buttel (1996) observed that scholars had tended to privilege one or the other of these categories, which may have resulted in some pertinent gaps in the literature. Environmental social scientific literature - itself intentionally environmental - has most prominently analyzed the intentionally environmental, that is, social movements, local struggles, conflicts, politics, values, justice, consciousness, modernization, management, and ethics related to the natural environment. In this paper, I focus on the unintentionally environmental, that is, the "normal workings" of our global industrial market society. While such normal workings have been addressed at the societal and global level in elaborate accounts (e.g. Foster 2000; Hornborg 2009; Moore 2014), these accounts have been interested in "the organization of labour to the exclusion of the practice of labour" (Schneider and McMichael 2010, 461 emphases in original). The unintentionally environmental at the practice level and at the level of subjectivity have not received sufficient attention, or so this essay argues and provides some ideas how to address them.

It is obvious for many that much of environmental change is produced by the habitual (see Kurz et al. 2015 for review), that "environmental concerns remain on the periphery of social life" (Macnaghten 2003, 63) and that for most of the people the human-tonature relationship is a highly mediated one (Dickson 2000; Stoner 2014; Weis 2013). Nonetheless, it seems that under a better part of the environmental social scientific and all of natural scientific - literature there lie individualist (Shove 2010), value-based (Stoner 2014), and cognitivist assumptions, albeit often latently (Arponen 2013, 2015). This is to see that more information, increased environmental consciousness, and 
"greener" values of individuals - as well as groups and masses - will do the job of saving the planet (Dickson 2000; Shove 2010). Another approach has been to shift the attention from the global environmental problems (which do not ring the bell) to the local environments that are already relevant for the people, like Macnaghten (2003) maneuvered. This, however, is to shy away from the global level destructive patterns the problem at hand. Focusing on environmental changes that are relevant for ordinary people would miss the majority of the global changes, most of which "are plainly remote from everyday life experience so that their reality is not apparent to ordinary citizens" (Yearley 2005, 315). Focusing on the subjectivity of the actor is not to embrace an individualist or a cognitivist stance, as will be clear.

The practice turn is argued to resolve the problems of individualism and exaggerated epistemic powers of actors (Arponen 2013) that Elizabeth Shove (2010) claimed to waste social scientific effort in helping to resolve environmental problems, a view agreed with here. While the practice stance takes practices as "the 'smallest unit' of social analysis" (Reckwitz 2002, 249) and hence effectively breaks away from the individualist undercurrent, this literature seems to be chiefly studying "a small-world of human action" (Arponen 2015, 6) and flat social practices such as showering, eating, cycling, driving, and consuming. Such dispersed practices, as Schatzki calls practices that "center around a single type of action" $(2002,88)$, cannot accommodate the highly differentiated masses carrying the (also) vertically organized complex practices. Moreover, they cannot easily reach far enough in spatial terms against the backdrop of the global market society (Arponen 2015). While Schatzkian practice theory underlies much of the consumption literature (Warde 2005; Shove 2010), some of Schatzki's central ideas of practices remain, at best, implicit in the literature. In particular, integrative practices - that is, "the more complex practices found in and constitutive of particular domains of social life" (Schatzki 1996, 98) - have not been adequately applied in the literature, even though this dichotomy has not remained unacknowledged (Warde 2005).

This paper belongs to phenomenological social scientific theorizing, which has been limitedly applied to environmental social sciences (Brown and Toadvine 2003; Williams and Parkman 2003). I lean heavily on Alfred Schütz's work (e.g. Schutz 1962, 1964) and build on Theodore Schatzki's practice theory (e.g. Schatzki 1996, 2010). It is noted that both Schutz's and Schatzki's work is complex and profound and cannot be addressed with much depth in a single essay. The style of the essay is therefore is to point to particular blind spots in the current literature and cast light upon avenues for future research. Below, I first address the "objective uneventfulness" of environmental changes. This is to explain how uneventful sociomaterial changes - an important part of all environmental changes - are produced by the very texture of social reality, that is, by social practices. Then, I scrutinize the "subjective irrelevance" of environmental changes by addressing the relevance structures and discuss the relation between the environmental layperson and environmental expert. I conclude by discussing implications.

\section{Objective uneventfulness}

Cumulative sociomaterial change is ubiquitous in the contemporary world that has crossed the planetary limits. Cumulative changes are products of the state of affairs, the established social world comprised of a mesh of orders and practices under a persistent process of naturalization (Bourdieu 1990), most aspects of which we tend to take for granted (Schutz 1962). While the Anthropocene pictures an essentially undifferentiated humanity producing the predicament of the planet, the concept of Capitalocene shifts 
the blame from individuals to the capitalist system, arguing that the planetary predicament is a structural problem (Moore 2014). Indeed, the late modernity entails an "unequal mass of actors" that, as Arponen explains, does "not share a sphere of life and thereby knowledge, beliefs, and the like about the broader system" $(2013,48)$. Such a view poses a conceptual dilemma for the practice literature that sees carriers of practices as dependent on shared assumptions. If the practice literature is to contribute to our understanding of the pertinently structural problem, it has to be able at least to conceptualize 1) practices that can accommodate highly differentiated carriers and 2) complex material arrangements. While one avenue for solving these problems could be to dig into the interrelatedness of practices, another path, chosen here, could be to see how activities of differentiated carriers of integrative practices "hang together" (Schatzki 1996,14). This is to build complexity into the concept of practice rather explain complexity with the interrelatedness of practices.

\subsection{Integrative and dispersed practices}

Adopting a practice view is to see practices as the texture of social reality: "the social world is first and foremost populated by diverse social practices" (Reckwitz 2002, 256). At its most basic level, a practice is "a temporally unfolding and spatially dispersed nexus of doings and sayings" (Schatzki 1996, 89). Yet, Schatzki (2002) argues that such concept of social practice is not enough to conceptualize social worlds, but we have to understand as well orders - arrangements of people, artifacts, and things. "Human coexistence," Schatzki writes, "transpires as and amid an elaborate, constantly evolving nexus of arranged things and organized activities" (2002, p. xi). Here, the concept of practice also involves Schatzki's orders and hence practices can even entail a highly complex arrangement of material artifacts, such as factories or infrastructures, that help to hang human activity together. Albeit acknowledged (Warde 2005), Schatzki's distinction between two ideal types of practices: dispersed and integrative (Schatzki 1996), has remained inadequately applied in literatures of consumption and sustainability. While Shove (2010) differentiates practices-as-entities and practices-asperformance based on Schatzki's writings, this is not the same distinction as that between simple and complex practices, which latter allow for conceptualizing large and hierarchically linked doings and sayings. I envision social practices in a continuum, one end of which accommodates integrative practices and the other end dispersed practices that course through integrative practices (Schatzki 2002).

Distinguishing between simple practices such as "describing, ordering, questioning, reporting, and examining" (Schatzki 2002, 88) and complex ones such as functionally, cognitively, and materially binding arrangements and activities of, say, an organization, is important in order to understand both the mundane and the - even globally orchestrated nature of human activity. Arrangements of things are especially important in integrative practices in which doings and sayings hang together prominently not only through understandings (as in dispersed practices), but also through explicit rules and teleoaffective structures that to Schatzki are "a range of normativized and hierarchically ordered ends, projects, and tasks, to varying degrees allied with normativized emotions and even moods" $(2002,80)$. Note, as Schatzki insists, that integrative practices should not be thought as "assemblages of dispersed practices, which are added together to form integrative ones" $(1996,99)$. The integrative end of the continuum should be seen to accommodate practices that entail even a highly complex arrangement of material artifacts. In addition to understandings, rules, and other cognitive structures, such material arrangements should be seen as a prominent source that makes the doings and sayings hang together. They are "mediatory objects" (Ginev 2014, 81) or "mediating 
artefacts" (Miettinen, Paavola, and Pohjola 2012, 9) that link subjective and objective spheres. Thus instead of bracketing such arrangements as the context, these arrangements could be seen as constitutive of practices. These sociomaterial arrangements of course also entail the natural environment constituting, together with the built environment, "the ontological structure of the pre-given world" (Schutz 1966, 125), "which we have to interpret in order to find our bearings within it and come to terms with it" (Schutz 1962, 10).

\subsection{Differentiated carriers of practices}

The vast and harmful material impacts produced by humanity are unevenly distributed between the center and periphery of the capitalist system; the corresponding modes of production involve highly unequal positions of individuals, groups, and societies alike (e.g. Hornborg 2009; Moore 2014). Therefore the pragmatic necessities (Schutz 1964) as well as cognitive issues such as knowledge, beliefs, values, and the like about this broader system sharply differ between people (Arponen 2013) even though they may be carriers within the same integrative practice. Understanding environmental predicament necessitates paying "attention to the role that power and social inequality play in shaping human/nonhuman interactions" (Pellow and Nyseth Brehm 2013, 229). Indeed, as Davide Nicolini put it, "practices and their association perform different and unequal social and material positions, so that to study practice is also the study of power in the making" $(2009,1394)$. If remaining close to the dispersed end of the practice continuum, it is difficult to see inequalities produced and maintained within and through practices. Seeing organizations within particular industries - or even supply chains as a whole - as integrative practices, we can fathom different carriers of such practices as located in particular points in hierarchical and geographical terms and with differentiated perspectives and knowledge of the activities that hang together not only through cognitive structures, but also through materiality (technology, other material artifacts, energy, and so on) and monetary relations and transactions (Hornborg 2009). Debt, in particular, has been a prominent binder of social actors that entails as well as produces inequalities (Gerber 2014).

The critique against individualist and small world approaches (Arponen 2015; Dickson 2000; Stoner 2014) urges us to understand the complex mediation of the sociomaterial structures through which our activities impact the natural and built environments. As Barnabas Dickson (2000) explained, most of us rarely act directly with the natural environment, but indirectly through others - most often through a chain of buyer-seller relations - who, most typically, are spatially distant from us. The concept of structural environmental burden underscores that "every good and service available to us presuppose the production processes behind their existence" (Arponen 2013, 50), which have already consumed matter-energy. Whereas it is certainly possible to impact the production of goods and services through consumer choices - particularly with regard to alimentation (Weis 2013) - due to the highly mediated nature of the market relations, such impacts should be seen as a very ineffective form of impacting the entire systems of production (Dickson 2000) and policies attempting to steer such choices even more so (Shove 2010). Furthermore, the mere existence of material infrastructure in and around, say, energy, transport, or hospitals inherently involves matter-energy, and individuals are most often not able to choose such infrastructures and can affect the existence of such infrastructures to a very small extent. 


\subsection{Practices and stuck and loose matter}

In order to explicate one very general level characteristic of some environmental changes - their cumulative nature - materiality involved in all practices is compressed to two ideal types, stuck and loose matter. Stuck matter refers to material artifacts tools or equipment (Miettinen et al. 2012) - involved in practices, as well as to highly complex material arrangements such as those involved in (the frames of) different infrastructures, that is, buildings, roads, hospitals, cables, and so on. Such materiality remains essentially unchanged through the particular moments of reproduction of practices that make use of it. While roads and other infrastructure wear out gradually, one car driving a road leaves it fundamentally intact, in the same way as one document leaves the cable through which it was downloaded essentially unchanged. Whereas the gradual decay of roads impacted by masses of cars through many years is an important phenomenon and can be seen as one type of cumulative change, the cumulative change focused on in this paper is such that requires an analysis of another category of materiality involved in practices: loose matter. Loose matter comes close to one of Schatzki's categories of materiality, that of "biological and physical flows that pass through practice-arrangement nexuses" (Schatzki 2010, 137). This refers to matterenergy, such as foods and fuels, but also the materiality worked on that might be "spontaneously provided by nature" or more commonly materiality "filtered through past labour" (Benton 1989, 65). Loose matter also has structural environmental burden, which in the case of oil products depends at least on the source, mode of production, and transport of oil. Loose matter is that materiality circulating in the metabolic accounts as material flows (Foster 2000).

Note that while stuck matter is functionally - instead of absolutely - stuck in the practice, thereby forging the characteristics of the practice, loose matter is indispensable for the running of the practice altogether. Farming practices, for instance, can make use of harvesters and other machines (stuck matter), but without the food, air, and water consumed by the farmer herself and energy from the sun, water, and nutrients (loose matter) that form the principal substance of the crops, farming practices cannot run at all. And if a harvester were employed, it would not run at all without fuel. Some stuck matter permits some types of activities that could not be done without them. Think of flying. Note that stuck and loose matter are defined only through their relation with practices and for this, the car is stuck matter within the practice of car commuting regardless of its loose nature in absolute terms when in motion. Also, some stuck matter is very briefly used within practices; think of disposable items. The boundaries between these relational categories are porous in the empirical reality and the flows involved depend to a large extent on the timescale we choose to study. It is intelligible to speak of stuck matter only with relation to particular moments of reproduction because over longer time periods, stuck matter flows in and out of practices creating, for instance, diverse flows of e-waste when new electronic devices replace the old. The categories of stuck and loose matter are very general; they are needed to conceptually clarify a cumulative type of sociomaterial change, which is a very general level process.

\subsection{Cumulative sociomaterial change}

Each and every practice requires inputs (or withdrawals) and produces outputs (or additions), therefore forming material flows of loose matter in between. ${ }^{1}$ Change

1 Additions can cumulate as more or less enduring stocks of loose matter, such as buildings, roads, and other infrastructures that function as stuck matter for a diverse set of practices. 
produced through reproduction of practices is cumulative in the sense that some loose sociomateriality is repeatedly added to or withdrawn from a system (Schnaiberg 1980), thus producing a buildup or drain - when it is not reused by another social practice or when natural processes do not absorb or renew such matter completely. Cumulative change is an aggregate phenomenon - it is born macro. Sociomaterial accumulation rarely makes sense at the unitary level (at the level of particular act of withdrawal or addition), but typically only when many unitary level recurrent acts are aggregated over time at some level. What is more, a discrete event at one level of observation - or in one lifeworld (Schutz 1964) - might be a continuation at more aggregate levels and objectively an uneventful occurrence. For instance, purchasing a car - or a bicycle - is a unitary level act that constitutes, together with many similar actions, practices around car (or bicycle) production and consumption. Such an act is often a major event for the purchaser. For the seller such an event is much less eventful - it is a mundane daily recursive activity. Furthermore, mere numbers mark it for the manufacturer, for a city planner, or for an economist. Zooming in and out of practices by foregrounding certain aspects and lifeworlds and bracketing others (Nicolini 2009) in synchronic or diachronic dimensions helps to understand how cumulative changes are produced and perceived.

Let us look another example. Building a mine or a factory is not a continuation or uneventful in any meaningful aspect at the local level, but instead often acutely contested and debated, especially when material grievances are present (Kröger 2014). There is, however, a sense in which such an event is a continuation of something. Such an investment can be seen as an embodiment of recipe knowledge of solving definite problems stating that the large-scale, centralized, and modernist units solve the problem of economic growth - and that of development - best (and exclusively). Applying such higher forms of knowledge (Schutz and Luckmann 1973) of an economist can be seen as making even global level activities hang together. Nevertheless, the most obvious objective uneventfulness of environmental change refers to that taking place after the construction of an industrial unit. When production begins it tends to be gradually institutionalized as a natural part of the sociohistorical constellation (Bourdieu 1990), particularly if there are no movements organized to contest it (Kröger 2014). Yet, such routinized production - even if it remains utterly unchanged - gradually alters its surrounding sociomateriality through recurrent withdrawals and additions. While in analytic terms we can analyze single practices, we have to understand that they "overlap, interweave, and also conflict" (Schatzki 2002, 88) with other integrative practices and hence cumulation is typically an aggregate cumulation of a set of different practices and hence seldom a linear pattern.

Accumulation inherently linked with loose matter differs from the typical referent of the term: capital accumulation. Cumulative change also does not refer to gradual change in the content of social practices and relations through "the processes of drift, layering, and conversion" (Moore 2011, 303), or to a continual evolution due to "changing circumstances, accumulating experience, bodily peculiarities, and shifts in the orders and practices that the actions engage or are part of" (Schatzki 2002, 242). It also does not refer to Hawley's cumulative change, which "constitutes growth of the system, a movement from small and simple to large and complex" $(1978,787)$. Needless to say that such non-cumulative (as understood here) changes in the contents and reproduction of practices are fundamentally important in the age of innovation. To recapitulate, accumulation here refers to a process characterized by recurrent acts "repetition of the same" (Schatzki 2002, 9) - through which pieces of sociomateriality are taken from or added to a particular sociomaterial system. It should be clear that such a change represents an ideal type (Schutz 1964), which is seldom found in its pure 
form in the empirical reality. Nonetheless, along with the increasing scale and scope of human material production, the normal workings of industrial societies require and consume enough loose matter to systematically create "rifts" disrupting the natural cycle of nutrients and materials at the planetary level (Foster 2000; Moore 2014). Accumulation - both drain and buildup - of sociomateriality takes place rather ubiquitously.

\subsection{Cognition, attention, and cumulative change}

How such accumulation and involved flows are structured has been given much attention in macro-sociological accounts. Much less effort has been allocated to understand the cognitions related with such change. Many scholars acknowledge the habitual nature of much of "environmentally consequential behavior" (Kurz et al. 2015). To be sure, all behavior is environmentally consequential; only some of it is intentionally so (Buttel 1996). Even though notions of ordinariness and mundane character of human behavior are not infrequently referred to (Arponen 2013; Kurz et al. 2015), a closer look at the cumulative sociomaterial change may reveal something new. All repeated activity tends to be habitualized and is in a process of becoming taken for granted (Berger and Luckmann 1966), resulting, as Bourdieu wrote, that "regularities inherent in an arbitrary condition ... tend to appear as necessary, even natural" (1990, 53-54). The ordinary (including the material arrangement) in a particular social situation is a result of past recurrent activity and tends to be taken for granted by the members of the corresponding social world. Instead of an event, the "repetition of the same" (Schatzki 2002,9 ) is an ordinary occurrence; it is the very source of the ordinary. Hence, not only environmental changes produced by the reproduction of social practices are cumulative, but also the recurrent additions and withdrawals gradually lose their objective eventfulness over time. Referring to uneventfulness is not to refer to something not taking place, but something taking place in a manner that not distinguished from what is apperceived as normal and to which we do not pay much attention. Even though no act can be repeated exactly in the same manner, we tend to typify experiences - there we go again (Berger and Luckmann 1966) - even if they are not exactly the same as previous ones.

In the same way that practices "always need to be drawn to the fore, made visible and turned into an epistemic object in order to enter discourse" (Nicolini 2009, 1392), cumulative changes also have to be drawn to the fore from their taken-for-grantedness in order to make them objects of attention - a work of environmental scientists, activists, journalists, and others. As we can observe only a fraction of environmental changes through our own sensory equipment, nearly all what we know of environmental changes is experts' observations made with the use of sensory equipment (Yearley 2005) and supplied to us through various types of media (Macnaghten 2003). Yet, "in an age that venerates instant spectacle," as Rob Nixon $(2011,6)$ characterizes the present time, media and scholars alike tend to pay attention to surprises rather than to what is uneventful. Cumulative changes then tend to be left "largely unobserved, undiagnosed and untreated" (Nixon 2011,6). No doubt the paramount cumulative change of our times, that of greenhouse gases in the atmosphere, is, regardless of all the bias (Boykoff 2011), massively discussed and debated in all media, but it should be understood, at most, as a tip of the iceberg in terms of the cumulative sociomaterial changes in the late modernity. In fact, the accumulation of greenhouse gases is an emergent aggregate of a diverse set of smaller cumulative changes that can be studied through particular integrative practices - those around industrial agriculture (Weis 2013) and fossil fuels in particular - that produce them. 
Land degradation, desertification, groundwater depletion, rural exodus, population growth, deforestation, urbanization, eutrophication, extinction of species, ecosystem destruction, and various other cumulative sociomaterial changes produced by the normal workings of the industrial-meat-eating-consumer civilization appear much less routinely in contemporary media (Almiron and Zoppeddu 2015; Boykoff 2011), partly because the paramount phenomenon - climate change - detracts attention from other phenomena (Crist 2007). How are such and other environmental changes experienced by different types of people?

\section{Subjective irrelevance}

The macro-sociological as well as practice level approaches have aimed to heave the political discussion around environmental predicament from individual (consumption) to more structural issues (Shove 2010) and attempted to collapse the agency-structure dualism. This essay is no different. Yet, whereas "social practice theory de-centres individuals from analyses" (Hargreaves 2011, 79) and practices are "the 'smallest unit' of social analysis" (Reckwitz 2002, 249), analyzing social practices should not inhibit looking as well at the subjectivity of those differentiated actors located in different positions in supply chains, geographical positions, different experiences, beliefs, and whatnot. Schatzki argues that "fuller understanding of the social nature of mind/action will yield deeper insight into the social character of the individual subject, the phenomenon from which all individualisms set out and to which all wholisms must at some point descend" (Schatzki 1996, 20). Schutz and Schatzki agree upon the importance of understanding how subjectivities are constituted within the life-world; that is, as a part of the mesh of orders and practices. Yet, taking seriously the critique against cognitivism (Arponen 2013, 2015), leaning on shared mentalities is problematic in the global market economy even if the institution of money - buyer-seller relations, debt relations, and so on - is imposing similar necessities on people through the very same relations that mediate our relationship to the natural environment (Dickson 2000; Gerber 2014; Hornborg 2009). Obviously, monetary relations impose distinct relevances on lenders and borrowers.

\subsection{Relevances and practical intelligibility}

What Schatzki calls practical intelligibility that "governs action by specifying what an actor does next in the continuous flow of activity" $(2002,75)$ comes close to Schutz's concept of relevance: "it is relevance to an individual which shapes the selectivity of his experience, that is, which determines what arouses his attention, where his attention leads him and how far it leads him" (Straßheim 2010, 1418). What is relevant is not the same as what is said to be important. What is relevant or practically intelligible makes sense to do and "also causes activity in the senses of formal and final-but not efficientcausality" (Schatzki 2002, 75 emphasis in original). Of pivotal importance is that relevances - or practical intelligibility - are constituted within the social and physical worlds as part of the mesh of orders and practices "where the realms of sociality and individual mentality/activity are at once organized and linked" (Schatzki 1996, 13). We cannot (rationally or with mere will of mind) choose our relevances because they are the result of our past experiences, which are always also, if not predominantly, pragmatically motivated and tied to our social and physical surroundings, which we can only limitedly modify. We can certainly try to and trying - itself an experience certainly changes something. Needless to say that the extent to which our activities are guided on issues beyond the pragmatic, that is, on epistemic or normativist determinations (Arponen 2015; Dickson 2000), greatly varies between different 
individuals, groups, and societies due to the very different sets of experiences people within have been exposed to.

\subsection{Structures of relevance}

Schutz distinguished structures of relevance through zones of reach, origination, and person types (Schutz 1964). First, Schutz identifies four zones of relevance that differ with regard to how we experience and are able to dominate these parts of reality. While the zone of primary relevance is "that part of the world within our reach which can be immediately observed by us and also at least partially dominated by us" (Schutz 1964, 124), in the other extreme there are zones of absolute irrelevance in which "no possible change occurring within them would - or so we believe - influence our objective at hand" (Schutz 1964, 125). Second, Schutz distinguishes between intrinsic and imposed relevances. In essence, the former type of relevance originates from our own activities and the latter is imposed by others' activities (Schutz 1964). Last, there are the ideal types of persons: the expert, the man on the street, and the well-informed. "The expert starts," Schutz writes, "from the assumption not only that the system of problems established within his field is relevant but that it is the only relevant system" (1964, 130). The world to the man on the street is "in first place not an object of our thought but a field of domination" (Schutz 1962, 227). The third type, the well-informed, gathers "as much as knowledge as possible of the origins and sources of the relevances actually or potentially imposed upon him" (Schutz 1964,130-131). These categories are ideal types and, as Schutz wrote "each of us in daily life is at any moment simultaneously expert, well-informed citizen, and man on the street, but in each case with respect to different provinces of knowledge" $(1964,123)$. Ideal types also provoke debate.

\subsection{Zones and environmental changes}

Even if real, most environmental changes "are plainly remote from everyday life experience" (Yearley 2005, 315) as the paramount reality of everyday life is the urban reality for the increasing part of the lay people and experts alike and accumulation typically (but not always) takes place in the edges of societies. Cumulative changes can certainly reach the pragmatic life of the urban lay people the epitome of which are the current water shortages in California and São Paulo. Typically, however, cumulative changes tend to "remain imperceptible to the senses, either because they are geographically remote, too vast or too minute in scale, or are played out across a time span that exceeds the instance of observation or even the physiological life of the human observer" (Nixon 2011, 15). Modern technology makes cumulative changes less visible even for the blue-collar specialists (Schnaiberg 1980); humans work with machines and machines work with loose matter, creating epistemic barriers between humans and loose matter and its accumulation. There is a non-identity between the objective and subjective dimensions of the environmental predicament (Stoner 2014). Not only do we not see the water needed to produce our beef and car - or our salad and bicycle for that matter - but we see even less the withdrawals and additions of such loose matter. In sum, most environmental changes take place outside the zone of primary relevance of the majority - of at least the urban populations - and hence, the environment is an intellectual problem (Foster and Holleman 2012) for most of us regardless of which type - lay people, well-informed, or expert - we belong to. This is to talk of the majority in the

Global North; obviously there are populations whose livelihoods are directly and drastically transformed by environmental changes, which belongs to the intentionally 
environmental phenomena that has been and should continue to be a prominent focus of environmental sociology.

\subsection{Origination and environmental changes}

The labor process has been alienated from the natural environment due to the concentration of the means of production on fewer and fewer hands and to the elaboration of increasingly sophisticated tools (Benton 1989; Foster 2000). Today the separation is close to complete in a substantial part of the populations. Hence the livelihood of a typical citizen - particularly in the Global North - is rarely directly dependent on the surrounding natural environment, so environmental changes have no or very little pragmatically bound - intrinsic - relevance for them. The functioning of the global industrial market economy conceals the impacts that changes in natural environments have on our livelihoods (Dickson 2000). The chains of buyer-seller relations are so long and complex that it is very difficult to fathom how changes impact our zone of primary relevance in any realistic terms, which does not inhibit making interpretations of such impacts. Others' observations and explications impose relevances of environmental changes on our subjective consciousness (Schutz 1964); "the global media are of major importance to how people understand and make sense of environmental issues" (Macnaghten 2003, 67). It is worthwhile to repeat that meanings so imposed to us are an intellectual issue (Foster and Holleman 2012) and whereas epistemic considerations (Arponen 2015) surely weigh in our behavior, they are often belittled by "the pragmatic necessities of the everyday life" (Berger and Luckmann 1966, 135).

The issue of awareness of environmental predicament has been a salient topic since the early environmental sociology (Buttel 1996; Rudel et al. 2011), yet analyzing the mere awareness is clearly not enough, as the value-action gap (Arponen 2015) implies. Much of human conduct is of everyday quality and even "our occupational activities," as Arponen writes, "are often daily recursive, very mundane and everyday activities, even when they require considerable concentration or other mental efforts" $(2013,50)$. Yet, it is so easy to embrace the assumption according to which "humans are rational, that humans make real choices, and that given enough time and education we will solve environmental problems," as Williams and Parkman note and continue that to "consider the reverse, that our consciousness is conditioned by taken-for-grantedness is a bitter pill for sure." $(2003,455)$ Continuing to assume rationally behaving individuals may be explainable by the fact that the environmental social scientific literature yet builds rather directly on natural scientific findings and takes the attached package of assumptions along.

\subsection{Environmental layperson and well-informed}

Typical persons tend to be assumed as in a deficit model, that is, as ignorant, but eager to acquire and accept (scientific) knowledge and "adapt to the changed epistemic circumstances" (Maranta et al. 2003, 162). Such a view comes close to Schutz's ideal type of well-informed citizen and assumes a subordination of human conduct to scientific mind, or at least to human rationality. Yet, there is a "need to think nonepistemically," as Arponen put it, "not in terms of what norms, values, beliefs and the like govern and drive human action, but how do human relate to each other in terms of their diverse activities" $(2015,13)$. Williams and Parkman expressed the problem bluntly: "Unless we better understand the pretheoretical and pragmatic nature of human consciousness, rational/scientific attempts to deal with large-scale 
environmental problems will fail" $(2003,449)$. Schutz argued that people tend to have a natural attitude in living their lives. The natural attitude towards life - which does not refer to epistemic attitudes toward particular issues in Shove's ABC model (2010) - is characterized as "a direct orientation towards things and not at all occupied with the critique of knowledge" (Moran 2013, 111), or as a typical attitude of everyday life "in which we have not theoretical but eminently practical interest" (Schutz 1962, 208). While certainly people do transcend such attitude of the man on the street and confront the reified appearances, pertinent phenomenological argument is that many of us do not do that most of the time. Furthermore, Schutz writes: "In all matters not connected with such practical purposes of immediate concern the man on the street accepts his sentiments and passions as guides" $(1964,122)$. The debate upon the existence of climate change seems to demonstrate that there are indeed plenty of men and women on the street. Whereas we may aim to be well-informed in some spheres of life, for instance, in terms of the food we eat, it is quite another issue how well we succeed to actually know the origins, impacts, and contents of our food in our particular material arrangement or even less to acquire and consume such food we deem healthy and ecological - insofar as we are determined to do so. It should be clear that such success is to a large extent not a question of individual treat, but a practice level issue both in epistemic dimension of inducing the determinations and in material dimension affording to do so. To what extent do the social realities and material arrangements structure our activities and how much can we deviate from them consciously? Another question to be studied empirically is whether, to what extent, and for how much of their time people in different parts of the world fit as referents to the above described ideal types.

\subsection{The environmental expert}

Much of environmental literature seems to assume environmental despoliation as a relevant issue for everyone insofar as they are aware of such issues (Stoner 2014). There is absolutely nothing wrong with being aware, conscious, and critical about environmental destruction. However, when it comes to analytically imposing such a stance on other people, things become more complicated. For Alfred Schütz, social scientific scholars have a theoretical attitude toward the social world; they interpret what is already pre-interpreted by the people in the social world. Such theoretical attitude is "that of a mere disinterested observer of the social world" (Schutz 1962, 36). Rather ironically, environmental social scientific scholars seem not to be disinterested observers with regard to the exacerbating impact humans have on their natural environment. Such concern is often the very reason for experts' research - they are highly interested observers. The disinterestedness refers to the fact that the scientist "is not involved in the actor's hopes and fears" and to the fact that scientists" "system of relevances differs from that of the interested parties" (Schutz 1962, 26). Indeed, "what is taken for granted in the biographical situation within the social world of the daily life may become questionable for the scientist, and vice versa; what seems to be of highest relevance on one level may become entirely irrelevant on the other" (Schutz 1962, 37). In an ideal case, expert's relevance system comprising of imposed relevances - that of environmental destruction - overrules all the other relevances, also the pragmatic relevances that the people in the social situation always have.

Certainly this provocative account simplifies things. The most prominent exception for the above account is of course that group of environmental experts who study particular cases of intentionally environmental phenomena through ethnomethodology or similar methods in which some - or even most - of the intrinsic relevances of the observer and the observed are shared. However, for the scholars studying the unintentionally 
environmental, the incommensurability between experts' imposed relevances and the layperson's intrinsic relevances may be an important lesson. If the claim that relevances are distinct is accepted, the subsequent question is how relevances can be imposed on others; that is, how to transform imposed relevances into intrinsic relevances. One lead could be to empirically analyze how perception of different types of messengers impacts our reception of relevances (e.g. Schutz 1964, 131-134). Such an analysis, however, remains in the epistemic sphere and we should, in tandem, study "how diverse activities and actors relate to each other" (Arponen 2015, 13) and the pragmatic necessities of

differentiated people as a part and in the midst of the mesh of orders and practices. How should we structure, in cognitive and material terms, the very experiences of the people so that their intrinsic relevances would be outright sustainable from the outset?

\section{Discussion and implications}

Criticism against both individualism and cognitivism in environmental social scientific literature is gaining momentum (e.g. Kurz et al. 2015; Shove 2010), possibly because social theory does not explain human activity correctly; the inactivity of the masses remains an anomaly (Arponen 2015; Beck 2010). This essay is part of such criticism. Alfred Schütz's work, as the foundation for social constructionism (Berger and Luckmann 1966), is easily seen as cognitivist and the focus on subjectivities as individualist stance. Yet, Schutz's work is both not individualist and not cognitivist since, as it should have become evident, the social and the physical world is fundamental in it in terms of the constitution of subjective relevances. Setting "the structure of the Lebenswelt as experienced by men in their natural attitude" (Schutz 1962,145) as one point of departure of environmental social scientific analysis and conducting sociological analyses of the reality of everyday life in which scholars would be "only tangentially interested in how this reality may appear in various theoretical perspectives to intellectuals" (Berger and Luckmann 1966,33) ought to be an important step toward an environmental sociology that aims to take seriously the pragmatic and non-epistemic dimension of human consciousness (Arponen 2015; Williams and Parkman 2003). In such an approach, individuals should be seen foremost as carriers of diverse social practices and their relevances formed through performing such practices (Bourdieu 1990; Shove 2010). It is a question of analyzing how the mesh of orders and practices induces relevances through structuring the experiences of the unequal mass of people hanging together (Schatzki 1996) as a part of the global system (Arponen 2013). The normal workings of industrialized market societies have been theorized most prominently through macro-sociological approaches asking, "what does all this mean for us, the scientific observer?" (Schutz 1964, 6). Such accounts are needed; they provide crucial insights at the systemic level and are indispensable in the political debates in which aggregate level issues are on the table. Yet they alone are not sufficient for the challenges of environmental sociology: "Like any complex problem, understanding the multiple dimensions of environmental change requires close and obsessive attention to detail from multiple perspectives" (Lockie 2015,1). Studying practices is not straightforward because they constitute "the scarcely notable background of everyday life" (Nicolini 2009, 1392) and our epistemic culture makes it cumbersome to analyze the pragmatic dimension of reality, which can be only limitedly accessed through what people say. While overt meanings acquired through surveys and other means might tell of awareness and consciousness, they hardly tell of relevances, which can only be accessed, as Bourdieu insisted, situating "oneself within 'real activity as such', that is, in the practical relation to the world" with its presences, urgencies, and preoccupations (1990, 52 emphasis in original). 
The natural environment is predominantly an issue at the intellectual level for the most of the people, particularly in the Global North - and hence a question of ethics, morality, and justice. Thus, the omission in this paper of the blossoming literatures of environmental justice and ethics should not be seen as a refutation to those fields. Quite the contrary, we should attempt to understand and change the epistemic sphere of humans (also) in terms of nature, and environmental rights and justice, for instance, are crucial in political struggles. But we should understand that knowledge about environmental destruction is an intellectual issue and that the intellectual sphere is frequently incommensurate with the pragmatic sphere; the pragmatic necessities, urgencies, preoccupations, and such (Bourdieu 1990; Schutz 1964) time and again overrun whatever moral, ethical, or other mental determinations we embrace. We might do differently than we have sworn to do simply because the latter might be exceedingly difficult to do or because the situation induced us to do something else. However, the main question not is whether one person decides to act in a particular manner, but how the relevances or practical intelligibilities of many people are structured through differential positions within practices, carriers of which can be changed if they are deemed not to fulfill their roles (Dickson 2000).

The mere awareness or consciousness of environmental problems is not enough; practices hardly change through increased intellectual awareness because they are foremost motivated by pragmatic concerns. Or are they? Has the scientific conception of the world replaced "the naïve, natural, pre-scientific approach to the world" (Moran $2013,106)$ ? How much has the natural attitude been replaced if at all and how much we are yet enslaved to our pragmatic needs? What really is relevant - genuinely important for people in their lifeworlds? How far can knowledge, ethics, and morality take us in the social reality in which virtually everything can be bought with money? How difficult in pragmatic terms should car commuting become so that people would not only start to think of changing, but also actually change their commuting habits? How to disrupt meat-eating habits that have massive environmental impacts? How well and to what extent can relevances be imposed through the symbolic and material spheres? How are the Internet, different media, and globalization impacting these spheres - and the paramount reality, our zone of primary relevance, itself? What are the major building blocks - and processes - creating the systems of relevance? These and other questions should be answered if we want to take up the challenge to understand what makes sense for people - as differentiated carriers of global integrative practices - to do (Schatzki 2002) and sufficient reasons to change what makes sense to do. How to make such challenges relevant for the environmental sociologists? 


\section{References}

Almiron, N., and M. Zoppeddu. 2015. "Eating Meat and Climate Change: The Media Blind Spot - A Study of Spanish and Italian Press Coverage." Environmental Communication 9(3): 307-325.

Arponen, V. P. J. 2013. "The Human Collective Causing of Environmental Problems and Theory of Collective Action: A Critique of Cognitivism." International Journal of Applied Philosophy 27(1): 47-65.

Arponen, V. P. J. 2015. "A Critique of an Epistemic Intellectual Culture: Cartesianism, Normativism and Modern Crises. "Journal for the Theory of Social Behaviour DOI: 10.1111/jtsb.12085

Beck, U. 2010. “Climate for Change, or How to Create a Green Modernity?” Theory, Culture \& Society 27 (2-3): 254-66.

Benton, T. 1989. "Marxism and Natural Limits: An Ecological Critique and

Reconstruction." New Left Review 70-107.

Berger, P. L., and T. Luckmann. 1966. The Social Construction of Knowledge: A Treatise in the Sociology of Knowledge. Garden City, NY: Doubleday.

Bourdieu, P. 1990. The Logic of Practice. Stanford University Press.

Boykoff, M. T. 2011. Who Speaks for the Climate? Cambridge: UK: Cambridge University Press.

Brown, C. S. and T. Toadvine, eds. 2003. Eco-Phenomenology: Back to the Earth Itself. Albany, NY: State University of New York Press.

Buttel, F. H. 1996. "Environmental and Resource Sociology: Theoretical Issues and Opportunities for Synthesis." Rural Sociology 61 (1): 56-76.

Crist, E. 2007. Beyond the Climate Crisis: A Critique of Climate Change Discourse. Telos 141: 29-55.

Dickson, B. 2000. The Ethicist Conception of Environmental Problems. Environmental Values 9: 127-152.

Foster, J. B. 2000. Marx's Ecology: Materialism and Nature. New York: Monthly Review Press.

Foster, J. B., and H. Holleman. 2012. "Weber and the Environment: Classical Foundations for a Postexemptionalist Sociology." American Journal of Sociology 117 (6): 1625-1673.

Gerber, J-F. 2014. "The role of rural indebtedness in the evolution of capitalism." Journal of Peasant Studies 41(5): 729-747.

Ginev, D. 2014. Social practices from the viewpoint of trans-subjective existentialism. European Journal of Social Theory 17(1): 77-94.

Hargreaves, T. 2011. "Practice-ing behaviour change: Applying social practice theory to pro-environmental behaviour change." Journal of Consumer Culture 11(1): 79-99.

Hawley, A. H. 1978. "The Presidential Address: Cumulative Change in Theory and in History." American Sociological Review 43 (6): 787-796.

Hornborg, A. 2009. "Zero-Sum World: Challenges in Conceptualizing Environmental Load Displacement and Ecologically Unequal Exchange in the World-System." International Journal of Comparative Sociology 2009 50(3-4): 237-262.

Kröger, M. 2014. Contentious Agency and Natural Resource Politics. London, UK:

Routledge.

Kurz, T., B. Gardner, B. Verplanken, and C. Abraham. 2015. WIREs Climate Change 6: 113-128.

Lockie, S. 2015. "Why environmental sociology?" Environmental Sociology 1 (1): 1-3. Macnaghten, P. 2003. "Embodying the environment in everyday life practices."

Sociological Review 51(1): 63-84.

Maranta, A., M. Guggenheim, P. Gisler, and K. Pohl. 2003. "The Reality of Experts and the Imagined Lay Person." Acta Sociologica 46 (2): 150-165. 
Miettinen, R., S. Paavola, and P. Pohjola. 2012. From Habituality to Change: Contribution of Activity Theory and Pragmatism to Practice Theories. Journal for the Theory of Social Behaviour 42(3): 345-360.

Moore, A. 2011. "The Eventfulness of Social Reproduction." Sociological Theory 29(4): 294-314.

Moore, J. W. 2014. "The Capitalocene, Part I: On the Nature and Origins of Our Ecological Crisis," unpublished paper, Fernand Braudel Center, Binghamton University.

Moran, D. 2013. "From the Natural Attitude to the Life-World." In Husserl's Ideen, edited by L. Embree and T. Nenon. Heidelberg, Germany: Springer.

Nicolini, D. 2009. "Zooming in and out: Studying Practices by Switching Theoretical Lenses and Trailing Connections." Organization Studies 30 (12): 1391-1418.

Nixon, R. 2011. Slow Violence and the Environmentalism of the Poor. Cambridge, MA: Harvard University Press.

Pellow, D. N., and H. Nyseth Brehm. 2013. "An Environmental Sociology for the TwentyFirst Century.” Annual Review of Sociology 39: 229-250.

Reckwitz, A. 2002. "The Status of the 'material' in Theories of Culture: From 'social Structure' to 'artefacts.'” Journal for the Theory of Social Behaviour 32 (2): 195-217. Rudel, T. K., J. T. Roberts, and J. Carmin. 2011. "Political Economy of the Environment." Annual Review of Sociology 37: 221-238.

Schatzki, T. R. 1996. Social Practices: A Wittgensteinian Approach to Human Activity and the Social. Cambridge, UK: Cambridge University Press.

Schatzki, T. R. 2002. The Site of the Social: A Philosophical Exploration of the Constitution of Social Life and Change. University Park, PA: Pennsylvania State University Press.

Schatzki, T. R. 2010. "Materiality and Social Life." Nature and Culture 5 (2): 123-149. Schnaiberg, A. 1980. Environment: From Surplus to Scarcity. Oxford, UK: Oxford University Press.

Schneider, M., and P. McMichael. 2010. "Deepening, and Repairing, the Metabolic Rift." Journal of Peasant Studies 37 (3): 461-484.

Schutz, A. 1962. Collected Papers, I: The Problem of Social Reality. The Hague, Holland: Martinus Nijhoff.

Schutz, A. 1964. Collected Papers II: Studies in Social Theory. The Hague, Holland:

Martinus Nijhoff.

Schutz, A., and T. Luckmann. 1973. The Structures of the Life-World, Volume 1. Evanston, IL: Northwestern University Press.

Shove, E. 2010. "Beyond the ABC: climate change policy and theories of social change." Environment and Planning A, 42: 1273-285.

Stoner, A. M. 2014. "Sociobiophysicality and the Necessity of Critical Theory: Moving beyond Prevailing Conceptions of Environmental Sociology in the USA." Critical Sociology 40(4): 621-642.

Straßheim, J. 2010. "Relevance Theories of Communication: Alfred Schutz in Dialogue with Sperber and Wilson." Journal of Pragmatics 42 (5): 1412-1441.

Warde, A. 2005. "Consumption and Theories of Practice." Journal of Consumer Culture 5 (2): 131-153.

Weis, T. 2013. The Ecological Hoofprint: The Global Burden of Industrial Livestock. London: Zed Books.

Williams, J. and S. Parkman. 2003. "On Humans and Environment: The Role of Consciousness in Environmental Problems." Human Studies 26 (4): 449-460.

Yearley, S. 2005. "The Sociology of the Environment and Nature." in The SAGE Handbook of Sociology, edited by C. Calhoun, C. Rojek, and B. S. Turner, 314-326. London: SAGE. 